\title{
Editorial
}

\section{Discrete and Dynamic Optimization Problems in Operations Management 2014}

\author{
Xiang Li, ${ }^{1}$ Ou Tang, ${ }^{2}$ Weihua Liu, ${ }^{3}$ and Xiaochen Sun ${ }^{4}$ \\ ${ }^{1}$ College of Economic and Social Development, Nankai University, Tianjin 300071, China \\ ${ }^{2}$ Department of Management and Engineering, Linköping University, 58183 Linköping, Sweden \\ ${ }^{3}$ School of Management, Tianjin University, Tianjin 300072, China \\ ${ }^{4}$ Department of Mathematics, School of Science, Tianjin University, Tianjin 300072, China
}

Correspondence should be addressed to Xiang Li; xiangli@nankai.edu.cn

Received 9 December 2014; Accepted 9 December 2014; Published 31 December 2014

Copyright (C) 2014 Xiang Li et al. This is an open access article distributed under the Creative Commons Attribution License, which permits unrestricted use, distribution, and reproduction in any medium, provided the original work is properly cited.

This special issue in 2014, in the spirit of its predecessor published in 2013, continues with the aim of presenting the original research and review articles on the latest theoretical, numerical, and practical achievements on discrete and dynamic optimization problems in operation management. The issue contains 18 papers as follows.

$\mathrm{X}$. Bao studies the influence of two types of overconfident behavior, overestimation, and overprecision, on decision of capacity recovery when power system's critical capacity is seriously damaged. A newsvendor model is used to prove that increasing regulatory punishment for electricity shortage and providing subsidy for capacity recovery are conducive measures to calibrate insufficient service level caused by an overconfident manager.

L. Geng et al. focus on the self-organization of supply chain with a MAS-based supply chain resilience model established. The local fitness function and neighborhood structure as well as interaction rules applicable to supply chain system are designed through viewing the enterprise as agent. It is found that there is agglomeration effect and SOC characteristic in supply chain and the evolution of supply chain is controlled by parameters of MAS.

$\mathrm{X}$. Li introduces a review paper which proposes some hot issues in the current research by examining the most related existing literature from the perspective of operations management. Some insights and future research directions in this field are generated.

Y. Li et al. investigate the actuarial models of defined contribution pension plan. Through assumptions and calculations, the expected replacement ratios of three different defined contribution pension plans are compared. Particularly, more significant considerable factors are put forward in the further cost and risk analyses. In order to get an assessment of current status, the paper finds a relationship between the replacement ratio and the pension investment rate using econometrics method.

Q.-C. Meng et al. model the centralization ordering problem of retailers who face stochastic demands when the suppliers offer free shipping, in which limited distributional information such as known mean, support, and some deviation measures of the random data is needed only. The optimal order strategies of retailers are explored based on the linear decision rule mainly for stochastic programming. Further, the core allocation is also presented among all retailers.

C. G. Monyei et al. examine the characterization of six oil wells and the allocation of gas considering limited and unlimited case. A successive application of modified artificial neural network (MANN) is presented combined with a mild intrusive genetic algorithm (MIGA) to the oil well characteristics.

J. Qin and W. Liu develop an EOQ model under trade credit financing with ramp type demand and the demand dependent production rate. Subsequently, the algorithms are proposed to decide the optimal replenishment cycle and the optimal order quantity for the retailer. Finally, the numerical analysis is demonstrated to illustrate the models and the sensitively analysis is carried out to give some managerial insights.

D.-L. Sheng and X. Rong analyze the mean-variance insurers with the return of premiums clauses to study the 
optimal time-consistent investment strategy for the DC pension merged with an annuity contract. Both accumulation phase before retirement and distribution phase after retirement are studied. The numerical analysis is also conducted.

L. Xia and H. Zhi study the Stackelberg game between a retailer and a manufacturer considering the cap and trade system and analyse the impact of system parameters on the participants' decision making. It is also shown that the sidepayment self-enforcing contract can resolve the arguments that the existing research overemphasizes on spontaneity of participation in side-payment contracts design.

W. Xue et al. establish the decision making model for a firm procuring multiple suppliers and spot markets. The suppliers are unreliable and provide different types of optiontype supply contracts which should be made before demand realization, while the spot market can only be used after demand realization and has both the price and liquidity risks. The optimal portfolio policy for the firm is developed with conditions to find the qualified suppliers.

L. Zhang et al. focus on optimal investment strategy for a dual risk model under the condition that the company can invest into a risk-free asset and a risky asset. The precommitted strategy and time-consistent strategy are compared with the following results: the former can make value function maximized at the original time $t=0$ and the latter strategy is time-consistent for the whole time horizon.

L. Zhang et al. establish a double objectives path optimization model with the consideration of carbon emissions cost and economy cost. The DNA-ant colony algorithm is used to solve the problem and its performance is shown to be better than that of the basic ant colony algorithm.

$H$. Zhang et al. propose the macroscopic model of air traffic flow in airport terminal area and carried out a series of simulation experiments with the NetLogo platform. Through both of the theoretical and practical discussions, the basic interrelationships and influential factors of air traffic flow characteristic parameters are revealed.

J.-H. Zhang et al. study a two-level and four-party supply chain consisting of a supplier, an e-commerce platform, the third-party logistics, and a demander. The existence of maximum profit of supply chain is obtained and the supply chain coordination issue is considered.

Y. Zhou et al. use an evolutionary ant colony algorithm based on RFID and knowledge refinement to solve the routing optimization problem of warehouse intelligent vehicle in complex environments. The algorithm is shown to be more effective than the traditional ant colony algorithm and genetic algorithm with higher convergence speed, and it can jump out of the U-type or V-type obstacle traps easily.

Some theoretical researches are also included in this special issue.

L. F. Lu et al. propose a new decomposition model based on accelerated proximal gradient method for packet-level traffic data and present the iterative scheme of the algorithm for network anomaly detection problem, which is termed as NAD-APG.

L. Shi et al. propose an approach to assess the gauge capability when a simple linear profile is used to reflect product quality. This method can simplify the problem when the measured values are multidimensioned. The example of spring measurement is presented in this paper which shows how to implement the proposed method.

L. Xu et al. introduce a new difference system from a differential competition system using different discrete methods. The paper gives theoretical analysis for local bifurcation of the fixed points and derives the conditions under which the local bifurcations such as flip occur at the fixed points. Furthermore, one- and two-dimensional diffusion systems are given when diffusion terms are added.

\author{
Xiang $\mathrm{Li}$ \\ Ou Tang \\ Weihua Liu \\ Xiaochen Sun
}




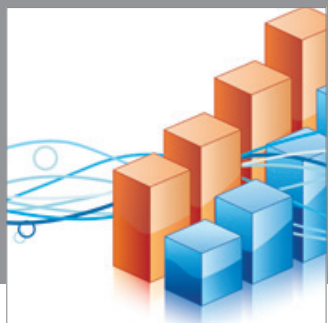

Advances in

Operations Research

mansans

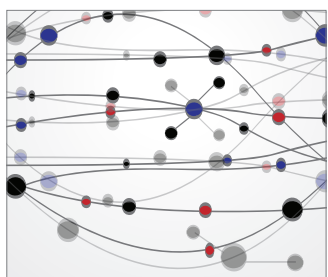

The Scientific World Journal
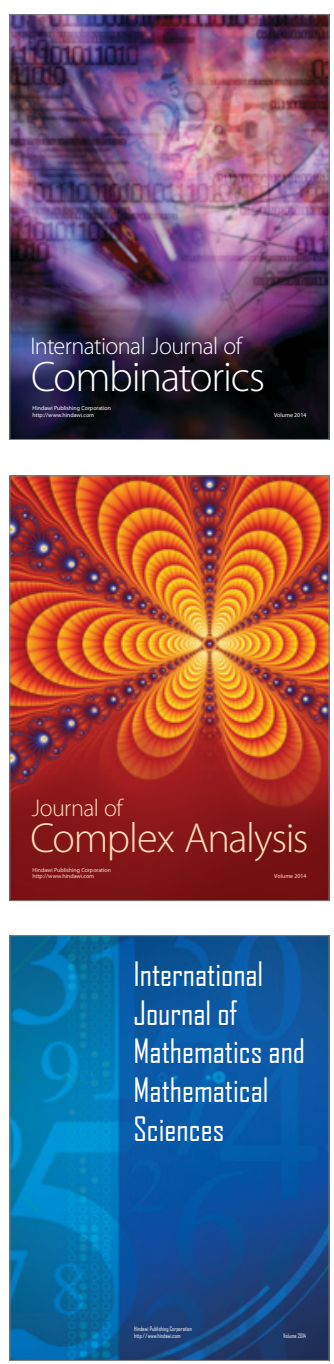
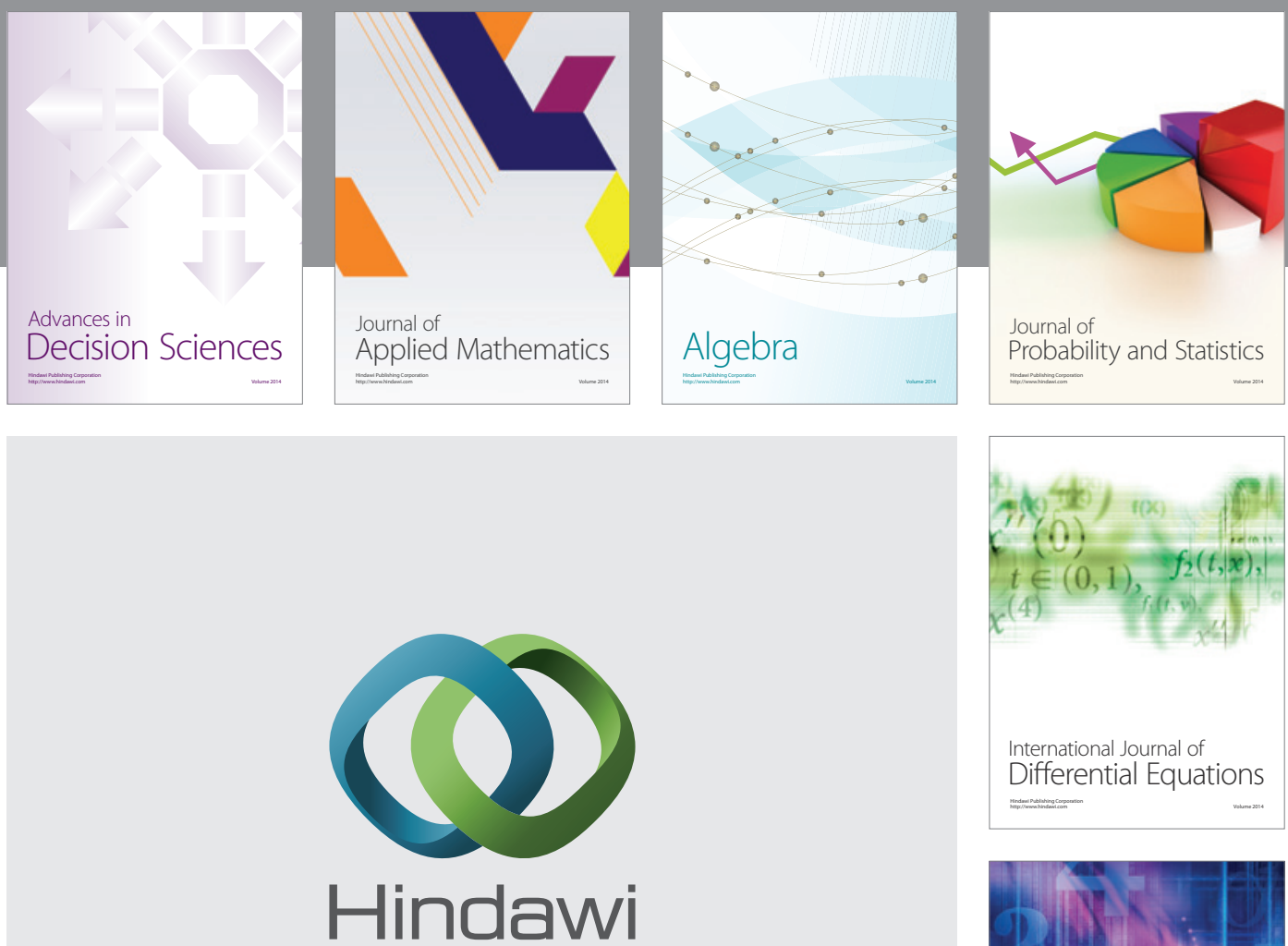

Submit your manuscripts at http://www.hindawi.com
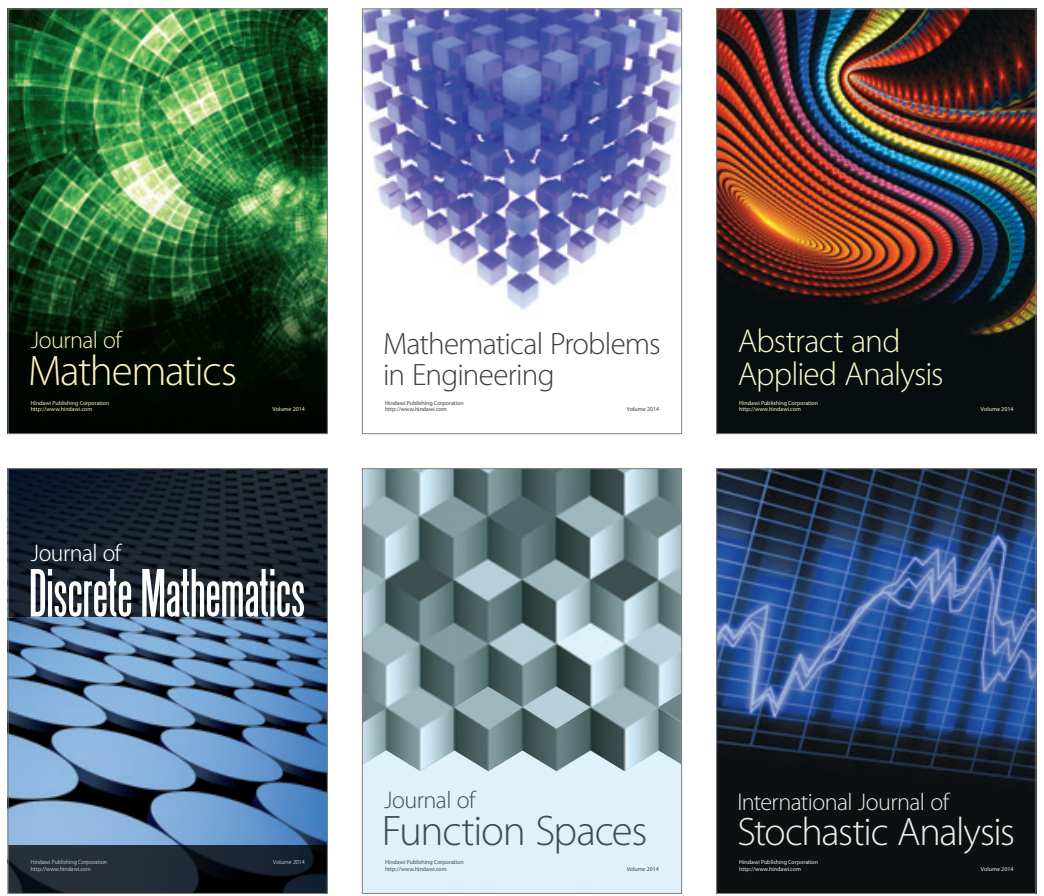

Journal of

Function Spaces

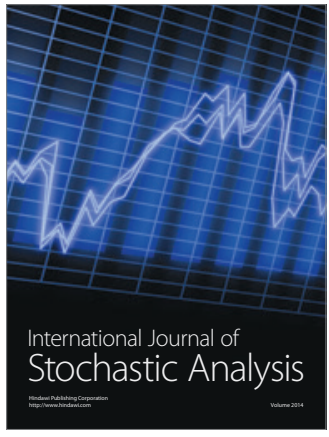

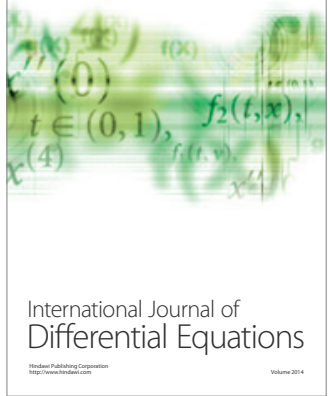
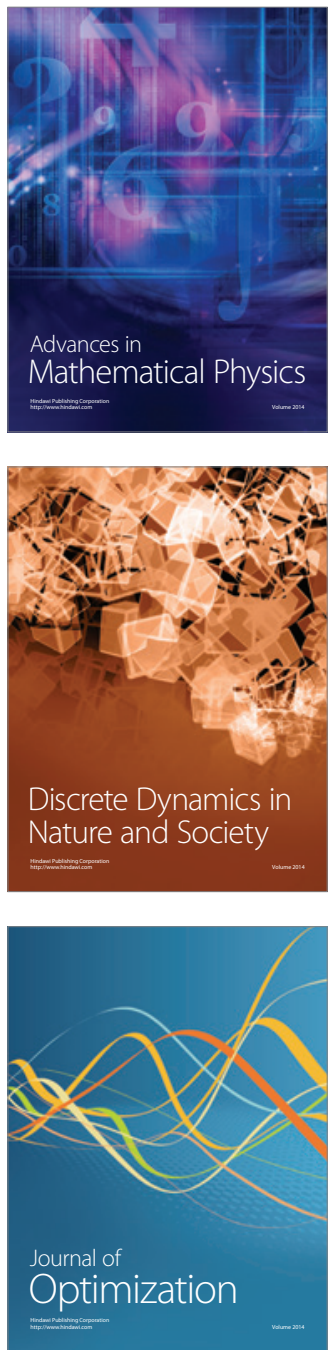